\title{
Construction of Exactly Solvable Ring-Shaped Potentials
}

\author{
Arup Bharali ${ }^{*}$, Ngangkham Nimai Singh ${ }^{2}$ \\ ${ }^{1}$ Bajali College, Pathsala-781325, Assam, India \\ ${ }^{2}$ Department of Physics, Gauhati University, Guwahati-781014, Assam, India \\ Email: *arup.brp@gmail.com
}

Received January 2, 2013; revised February 1, 2013; accepted February 10, 2013

Copyright (C) 2013 Arup Bharali, Ngangkham Nimai Singh. This is an open access article distributed under the Creative Commons Attribution License, which permits unrestricted use, distribution, and reproduction in any medium, provided the original work is properly cited.

\begin{abstract}
We propose a method for construction of exactly solvable ring-shaped potentials where the linear homogeneous secondorder differential equation satisfied by special function is subjected to the extended transformation comprising a coordinate transformation and a functional transformation to retrieve the standard Schrödinger polar angle equation form in non-relativistic quantum mechanics. By invoking plausible ansatze, exactly solvable ring-shaped potentials and corresponding angular wave functions are constructed. The method is illustrated using Jacobi and hypergeometric polynomials and the wave functions for the constructed ring-shaped potentials are normalized.
\end{abstract}

Keywords: Schrödinger Equation; Extended Transformation; Special Function; Ring-Shaped Potential; Normalization

\section{Introduction}

Generation/construction of exactly solvable quantum mechanical potentials is an important topic of fundamental research; as such type of research always incorporates new ideas and/or mathematical techniques to quantum mechanics. Again, exactly solvable potentials are essential for the successful implementation of approximate methods in the study of practical quantum systems. The study of quantum systems with non-central potentials is an upcoming field of research for the theoreticians. The quantum systems with non-central potentials have been studied extensively in quantum chemistry and nuclear physics in the context of organic molecules and deformed nuclei respectively in non-relativistic regime. Different methods applied by various authors to obtain exact solutions of Schrödinger equation for bound states with noncentral potentials, are the factorization method [1], the standard approach [2], the path integral representation [3], the Nikiforov-Uvarov method [4-6], the supersymmetric approach [7], etc. Here, we apply a method for construction of exactly solvable ring-shaped potentials starting from the linear homogeneous second-order differential equations satisfied by the special functions. We perform the extended transformation [8] consisting of a coordinate trans-

"Corresponding author. formation and a functional transformation on secondorder differential equation for a particular special function to mould the differential equation to the standard Schrödinger polar angle equation form. The coordinate transformation is the basic transformation required to change the characteristics of the differential equation, while the functional transformation is essential to retrieve the Schrödinger polar angle equation form. By invoking suitable ansatze, exactly solvable ring-shaped potentials and their angular wave functions are constructed. The special functions are found to be the multiplicative factors in the angular wave functions and the wave functions are normalized. In standard literature, the Schrödinger equation for a specific exactly solvable ring-shaped potential is solved by transforming it to a differential equation satisfied by a particular special function [1,2,9], but the ethics of the present work is not to solve Schrödinger equation to obtain wave functions for a particular ring-shaped potential, but to construct exactly solvable ring-shaped potentials as well as their angular wave functions starting from secondorder differential equations satisfied by special functions. The fundamental nature of the adopted method for transforming a solvable differential equation to a particular type bearing some physical significance would have a wide range of applicability not only in quantum mechanics but in other branches of science also.

The plan of the paper is as follows: in Section 2, for- 
malism of the method is discussed, construction of exactly solved ring-shaped potentials using the method is demonstrated in Section 3 and concluding remarks are included in Section 4.

\section{Formalism}

In the method, we start with a linear homogeneous second-order differential equation satisfied by a particular special function, on which we perform the extended transformation to generate the Schrödinger polar angle equation form and by invoking a suitable ansatz, the Schrödinger polar angle equation for a ring-shaped potential is retrieved from the generated equation. We now consider the following linear homogeneous second-order differential equation satisfied by a special function $F(z)$

$$
F^{\prime \prime}(z)+P(z) F^{\prime}(z)+Q(z) F(z)=0,
$$

where $P(z)$ and $Q(z)$ are well defined for the special function $F(z)$. We perform the extended transformation [8] consisting of a coordinate transformation and a functional transformation as follows

$$
z \rightarrow g(\theta)
$$

and

$$
\chi(\theta)=f(\theta)^{-1} F[g(\theta)]
$$

on the above differential Equation (1) yielding

$$
\begin{aligned}
& \chi^{\prime \prime}(\theta)+\left(\frac{\mathrm{d}}{\mathrm{d} \theta} \ln \frac{f^{2} \exp \int P(g) \mathrm{d} g}{g^{\prime}}\right) \chi^{\prime}(\theta) \\
& +\left(\frac{\mathrm{d}}{\mathrm{d} \theta} \ln f\right)\left(\frac{\mathrm{d}}{\mathrm{d} \theta} \ln \frac{f^{\prime} \exp \int P(g) \mathrm{d} g}{g^{\prime}}\right) \chi(\theta)=0 .
\end{aligned}
$$

The transformation function $g(\theta)$ is a smooth differentiable function of at least class $C^{2}$ and $f(\theta)^{-1}$ is the modulating function required to mold the above equation to the standard Schrödinger polar angle equation form. We make the coefficient of the first-order derivative equal to $\cot \theta$, fixing the functional form of $f(\theta)$ as

$$
f(\theta)=C_{N} \sqrt{g(\theta) \sin \theta} \exp \left[-\frac{1}{2} \int P(g) \mathrm{d} g\right],
$$

which changes Equation (4) to

$$
\begin{aligned}
& \chi^{\prime \prime}(\theta)+\cot \theta \chi^{\prime}(\theta)+\left\{\frac{1}{2}\{g, \theta\}-\frac{1}{4}\left(1+\csc ^{2} \theta\right)\right. \\
& \left.+\left[g^{\prime}(\theta)\right]^{2}\left[Q(g)-\frac{1}{2} P^{\prime}(g)-\frac{1}{4}[P(g)]^{2}\right]\right\} \chi(\theta)=0
\end{aligned}
$$

Where the Schwartzian derivative $\{g, \theta\}=g^{\prime \prime \prime} / g^{\prime}-(3 / 2)\left(g^{\prime \prime} / g^{\prime}\right)^{2}$ and $C_{N}$ in Equation (5) will act as normalization constant.

To retrieve Schrödinger polar angle equation, the following identity must be prescribed

$$
\begin{aligned}
& (\lambda+1 / 4)-\frac{m^{2}-1 / 4}{\sin ^{2} \theta}-V(\theta) \\
& =\frac{1}{2}\{g, \theta\}+g^{\prime}\left[Q(g)-\frac{1}{2} P^{\prime}(g)-\frac{1}{4} P(g)^{2}\right],
\end{aligned}
$$

where $\lambda=l(l+1)$ and $l$ and $m$ are the orbital and magnetic quantum numbers. In presence of a central potential, the admissible values for $l$ are $0,1,2, \cdots, n$ and for $m$ are $0, \pm 1, \pm 2, \cdots, \pm l$ where $n$ is the principal quantum number. But $l$ needs to be redefined [9] for a quantum system with central potential plus a polar angle dependent ring-shaped potential.

By putting first the expressions for $P(g)$ and $Q(g)$ defining a special function $F(g)$ in the above equation (7) and then by invoking an ansatz that there should be at least one constant term in right side of the above Equation (7), the functional form of the transformation function $g(\theta)$ is specified. Putting $g(\theta)$ in the Equation (7) again, one can in principle construct exactly solvable ring-shaped potential $V(\theta)$. The chosen term, of course, should be integrable to obtain $\theta=g(\theta)$ which again to be invert- ible to obtain the functional form of the transformation function $g(\theta)$. Using the expressions for $P(g), Q(g)$ and $g(\theta)$ in Equations (3) and (5), the angular wave function for the constructed ring-shaped potential is obtained as

$$
\begin{aligned}
\chi(\theta)= & C_{N}\left[g^{\prime}(\theta) \sin \theta\right]^{-1 / 2} \\
& \times \exp \left[\frac{1}{2} \int P(g) \mathrm{d} g\right] F[g(\theta)] .
\end{aligned}
$$

The normalization constant $C_{N}$ is evaluated by using the following normalization condition for $\chi(\theta)$ as

$$
I(0, \pi)=\int_{0}^{\pi}|\chi(\theta)|^{2} \sin \theta \mathrm{d} \theta=\text { Finite } .
$$

\section{Application}

We choose Jacobi polynomials and hypergeometric function to construct exactly solvable ring-shaped potentials using the method. Gegenbauer, Chebyshev and Legendre polynomials can be obtained as special cases from Jacobi polynomials. Again Jacobi polynomials can be obtained from hypergeometric function as special cases and the same holds for the generalized Laguerre, Hermite polynomials and the confluent hypergeometric function [10].

\subsection{Using Jacobi Polynomials}

The differential equation [10] satisfied by Jacobi Polynomial $F(z)=P_{n}^{(\alpha, \beta)}(z)$ is

$$
\begin{aligned}
& \left(1-z^{2}\right) F^{\prime \prime}(z)+[\beta-\alpha-(\alpha+\beta+2) z] F^{\prime}(z) \\
& +n(n+\alpha+\beta+1) F(z)=0,
\end{aligned}
$$


for which $P(g)=[\beta-\alpha-(\alpha+\beta+2) z] /\left(1-z^{2}\right)$ and $Q(g)=n(n+\alpha+\beta+1) /\left(1-z^{2}\right)$, the Equation (7) becomes

$$
\begin{aligned}
& (\lambda+1 / 4)-\frac{m^{2}-1 / 4}{\sin ^{2} \theta}-V(\theta) \\
& =\frac{1}{2}\{g, \theta\}+\left[n(n+\alpha+\beta+1)-\frac{1}{2}(\alpha+\beta+2)\right] \frac{g^{\prime 2}}{1-g^{2}} \\
& +\frac{1}{2}\left(\beta^{2}-\alpha^{2}\right) \frac{g g^{\prime 2}}{\left(1-g^{2}\right)^{2}} \\
& +\left[(\alpha+\beta+2)-\frac{1}{4}(\beta-\alpha)^{2}\right] \frac{g^{\prime 2}}{\left(1-g^{2}\right)^{2}} \\
& \quad-\frac{1}{4}(\alpha+\beta+2)^{2} \frac{g^{2} g^{\prime 2}}{\left(1-g^{2}\right)^{2}} .
\end{aligned}
$$

Introducing the ansatz, we choose the second term in R.H.S. of the above equation as a constant independent of $\theta$. We suppose that

$$
\frac{g^{\prime 2}}{1-g^{2}}=C^{2}(\text { a constant })
$$

which specifies the functional form of $g(\theta)$ as

$$
g(\theta)=\sin (C \theta+\delta) .
$$

Selecting $\delta=\pi / 2$ and $C=1$ to satisfy the local property of the transformation function $g(\pi / 2)=0$, the above Equation (11) gives us the exactly solvable Makarov ringshaped potential $[7,9]$ as

$$
V(\theta) \mapsto V_{1}(\theta)=B_{1} \frac{\cos \theta}{\sin ^{2} \theta}
$$

and $l=-\frac{1}{2} \pm \sqrt{n(n+\alpha+\beta+1)+\frac{1}{4}(\alpha+\beta+1)^{2}}$, where $\alpha=\sqrt{m^{2}+B_{1}}$ and $\beta=\sqrt{m^{2}-B_{1}}$.

Using Equations (13) and expressions of $P$ and $Q$ for Equation (10) in (8), the angular wave functions corresponding to $V_{1}(\theta)$ are

$$
\chi(\theta)=C_{N}\left(\sin \frac{\theta}{2}\right)^{\alpha}\left(\cos \frac{\theta}{2}\right)^{\beta} P_{n}^{(\alpha, \beta)}(\cos \theta),
$$

while normalization constant $C_{N}$ is evaluated by using Equation (9) and orthogonality relation [10] for Jacobi polynomials

$$
\begin{aligned}
& \int_{-1}^{+1}(1-x)^{\alpha}(1+x)^{\beta}\left|P_{n}^{(\alpha, \beta)}(x)\right|^{2} \mathrm{~d} x \\
& =\frac{2^{\alpha+\beta+1} \Gamma(n+\alpha+1) \Gamma(n+\beta+1)}{(2 n+\alpha+\beta+1) n ! \Gamma(n+\alpha+\beta+1)},
\end{aligned}
$$

where $\alpha>-1$ and $\beta>-1$ and the constant is found to be

$$
C_{N}=\sqrt{\frac{2^{\alpha+\beta-1}(2 n+\alpha+\beta+1) n ! \Gamma(n+\alpha+\beta+1)}{\Gamma(n+\alpha+1) \Gamma(n+\beta+1)}} .
$$

Again, if the fourth term in R.H.S. of the Equation (11) is taken as a constant such that

$$
\frac{g^{\prime 2}}{\left(1-g^{2}\right)^{2}}=-C^{2}
$$

the transformation function $g(\theta)$ becomes

$$
g(\theta)=-\mathrm{i} \cot (C \theta+\delta) \text {. }
$$

To satisfy the local property $g(\pi / 2)=0$, we choose $C=1$ and $\delta=0$ and the exactly solvable ring-shaped potential [11] is found to be

$$
V(\theta) \mapsto V_{2}(\theta)=B_{2} \frac{\cos \theta}{\sin \theta}
$$

with

$$
m=\sqrt{n\left(n+\alpha+\alpha^{*}+1\right)-\left(\alpha+\alpha^{*}+2\right)\left(\alpha+\alpha^{*}+4\right) / 4+1 / 4}
$$

and $\alpha=\sqrt{(l+1 / 2)^{2}+\mathrm{i} B_{2}}$.

The angular wave functions for $V_{2}(\theta)$ are obtained by using Equation (18) and $P$ and $Q$ of Equation (10) in (8) as

$$
\begin{aligned}
\chi(\theta)= & C_{N}(\csc \theta)^{\left(\alpha+\alpha^{*}+1\right) / 2} \exp \left[\mathrm{i}\left(\alpha^{*}-\alpha\right) \theta / 2\right] \\
& \times P_{n}^{\left(\alpha, \alpha^{*}\right)}(-\mathrm{i} \cot \theta)
\end{aligned}
$$

and the normalization constant is calculated by using normalization condition as Equation (9) and orthogonality relation [11]

$$
\begin{aligned}
& \int_{-\infty}^{+\infty}\left(1+x^{2}\right)^{\left(\alpha+\alpha^{*}+1\right) / 2} \exp \left[-\mathrm{i}\left(\alpha-\alpha^{*}\right) \tan ^{-1} x\right] \\
& \times\left|P_{n}^{\left(\alpha, \alpha^{*}\right)}(\mathrm{i} x)\right|^{2} \mathrm{~d} x \\
& =\frac{\left[\Gamma(n+\alpha+1) \Gamma\left(n+\alpha^{*}+1\right)\right]}{\left[2^{\left(\alpha+\alpha^{*}+1\right)}\left(2 n+\alpha+\alpha^{*}+1\right) n ! \Gamma\left(n+\alpha+\alpha^{*}+1\right)\right]},
\end{aligned}
$$

and is found to be

$$
C_{N}=\sqrt{\frac{2^{\left(\alpha+\alpha^{*}+1\right)}\left(2 n+\alpha+\alpha^{*}+1\right) n ! \Gamma\left(n+\alpha+\alpha^{*}+1\right)}{\Gamma(n+\alpha+1) \Gamma\left(n+\alpha^{*}+1\right)}}
$$

Though $\frac{g g^{\prime 2}}{\left(1-g^{2}\right)^{2}}$ in the third term is integrable to have $\theta=g(\theta)$, but it is not invertible and hence it cannot be used to construct ring-shaped potential. Taking the last 
term as a constant with the choice $\frac{g^{2} g^{\prime 2}}{\left(1-g^{2}\right)^{2}}$ equal to $C^{2}$ and $-C^{2}$, the transformation functions respectively come out as $g(\theta)=\sqrt{1-\exp [-\mathrm{i} 2(C \theta+\delta)]}$ and $g(\theta)=\sqrt{1-\exp [-2(C \theta+\delta)]}$, yielding ring-shaped potentials not for physical interest.

\subsection{Using Hypergeometric Function}

The differential equation [10] for hypergeometric function $F(\theta)={ }_{2} F_{1}(\alpha, \beta ; \gamma ; z)$

$$
\begin{aligned}
& z(1-z) F^{\prime \prime}(z)+[\gamma-(\alpha+\beta+1) z] F^{\prime}(z) \\
& -\alpha \beta F(z)=0
\end{aligned}
$$

for which $P(z)=[\gamma-(\alpha+\beta+1) z] / z(1-z)$ and $Q(z)=-\alpha \beta / z(1-z)$ and the equation (7) becomes

$$
\begin{aligned}
& (\lambda+1 / 4)-\frac{m^{2}-1 / 4}{\sin ^{2} \theta}-V(\theta) \\
& =\frac{1}{2}\{g, \theta\}-\frac{1}{4}\left(\delta^{2}+2 \delta\right) \frac{g^{\prime 2}}{(1-g)^{2}} \\
& \quad-\frac{1}{2}(2 \alpha \beta+\gamma \delta) \frac{g^{\prime 2}}{g(1-g)}-\frac{1}{4}\left(\gamma^{2}-2 \gamma\right) \frac{g^{\prime 2}}{g^{2}},
\end{aligned}
$$

where $\delta=\gamma-(\alpha+\beta+1)$.

Introducing the ansatz, we select the third term as a constant such that

$$
\frac{g^{\prime 2}}{g(1-g)}=4 C^{2},
$$

which yields the transformation function $g(\theta)$ as

$$
g(\theta)=\cos ^{2}(C \theta+\varepsilon) .
$$

Choosing $\varepsilon=0$ and $C=1$ so that $g(\pi / 2)=0$, the constructed ring-shaped potential becomes

$$
V(\theta) \mapsto V_{3}(\theta)=\frac{B_{3}}{\cos ^{2} \theta}
$$

and the angular wave functions corresponding to $V_{3}(\theta)$ are obtained by using expressions for $P$ and $Q$ and Equation (25) in (8) as

$$
\begin{aligned}
\chi(\theta)= & C_{N}(\sin \theta)^{\alpha+\beta-\gamma}(\cos \theta)^{\gamma-\frac{1}{2}} \\
& \times{ }_{2} F_{1}\left(\alpha, \beta ; \gamma ; \cos ^{2} \theta\right),
\end{aligned}
$$

where $\alpha=\left(3 / 2+\sqrt{B_{3}+1 / 4}+l-m\right) / 2=-n$,

$\beta=\left(1 / 2+\sqrt{B_{3}+1 / 4}-l-m\right) / 2$ and

$\gamma=1 \pm \sqrt{B_{3}+1 / 4}=\alpha+\beta+m$.

The normalization constant is determined by using
Equation (9) and orthogonality relation [9]

$$
\begin{aligned}
& \int_{0}^{1} x^{\gamma-1}(1-x)^{\alpha-\gamma}\left|{ }_{2} F_{1}(-n, \alpha+n ; \gamma ; x)\right|^{2} \mathrm{~d} x \\
& =\frac{n ! \Gamma(n+\alpha-\gamma+1)(\Gamma \gamma)^{2}}{(\alpha+2 n) \Gamma(n+\alpha) \Gamma(\gamma+n)}
\end{aligned}
$$

and it is found to be

$$
C_{N}=\frac{1}{\Gamma \gamma} \sqrt{\frac{\Gamma \beta \Gamma(\gamma+n)(\beta+n)}{\Gamma(\beta-\gamma+1) n !}} .
$$

Again, choosing $\varepsilon=0$ and $C=1 / 2$ in Equation (25), so that $g(\pi)=0$, the constructed ring potential is found to be again Makarov ring-shaped potential given by the Equation (14) for which the angular wave functions become

$$
\begin{aligned}
\chi(\theta)= & C_{N}\left(\sin \frac{\theta}{2}\right)^{\alpha+\beta-\gamma}\left(\cos \frac{\theta}{2}\right)^{\gamma-1} \\
& \times{ }_{2} F_{1}\left(\alpha, \beta ; \gamma ; \cos ^{2} \frac{\theta}{2}\right)
\end{aligned}
$$

where $\alpha=1+l-\sigma / 2=-n, \beta=-l-\sigma / 2$, $\gamma=1+B_{1} / \sigma-\sigma / 2$ and $\sigma= \pm \sqrt{2\left(m^{2} \pm \sqrt{m^{4}-B_{1}^{2}}\right)}$ and normalization constant

$$
C_{N}=\frac{1}{\Gamma \gamma} \sqrt{\frac{\Gamma \beta \Gamma(\gamma+n)(\beta+n)}{\Gamma(\beta-\gamma+1) n !}}
$$

Picking the second and third terms separately as constants, we cannot construct any ring-shaped potentials that will be functions of some trigonometric functions.

\section{Conclusion}

We present a method for construction of exactly solvable ring-shaped potentials with consideration of special functions in the framework of non-relativistic quantum mechanics. The method is realizable only for the implication of the extended transformation which is a coordinate transformation supplemented by a functional transformation. The extended transformation is performed on the linear homogeneous second-order differential equation satisfied by a particular special function to retrieve the Schrödinger polar equation form and by invoking plausible ansatze, exactly solvable ring-shaped potentials are constructed. For implementation of the method, we choose Jacobi polynomial and hypergeometric function as special functions to construct new (in Equation (26)) as well as already known exactly solvable ring-shaped potentials. The angular wave functions corresponding to the constructed potentials are normalized and also analytically verified. Though orbital quantum number takes the values $0,1,2,3, \cdots$ in presence of central potential, the same 
quantum number will depend on both magnetic quantum number and characteristic constants in presence of ringshaped potentials. Again, some unphysical potential are also come up in the calculations and new technique is required to make them physical. Laguerre, Hermite, Romanovski polynomials, etc. can also be utilized in the method to construct ring-shaped potentials. The proposed method has also the capability for construction of central potentials in non-relativistic regime and the essence of the method of converting a soluble differential equation to another differential equation of practical interest can be extrapolated to other branches of physics.

\section{REFERENCES}

[1] J. Sadeghi and B. Pourhassan, "Exact Solution of the Non-Central Modified Kratzer Plus a Ring-Shaped Like Potential by the Factorization Method," Electronic Journal of Theoretical Physics, Vol. 5, No. 17, 2008, pp. 193202.

[2] M. Zhang, B. An and H. Guo-Qing, "Exact Solutions of a New Coulomb Ring-Shaped Potential," Journal of Mathematical Chemistry, Vol. 48, No. 4, 2010, pp. 876-882. doi:10.1007/s10910-010-9715-1

[3] L. Chetouani, L. Guechi and T. F. Hammann, "Exact Path Integral for the Ring Potential," Physics Letters A, Vol. 125, No. 6-7, 1987, pp. 277-281.

[4] M. Zhang, G. Sun and S. Dong, "Exactly Complete Solutions of the Schrödinger Equation with a Spherically Har- monic Oscillatory Ring-Shaped Potential," Physics Letters A, Vol. 374, No. 5, 2010, pp. 704-708. doi:10.1016/i.physleta.2009.11.072

[5] S. Ikhdair, "Exact Solutions of the D-Dimensional Schrödinger Equation for a Pseudo-Coulomb Potential Plus RingShaped Potential," Chinese Journal of Physics, Vol. 46, No. 3, 2008, pp. 291-306.

[6] A. Antia, N. Ikot and L. Akpatio, "Exact Solutions of the Schrödinger Equation with Manning-Rosen Potential Plus a Ring-Shaped Like Potential by Nikiforov-Uvarov Method," European Journal of Scientific Research, Vol. 45, No. 1, 2010 pp. 107-118.

[7] B. Gonul and I. Zorba, "Supersymmetric Solutions of NonCentral Potentials," Physics Letters A, Vol. 269, No. 2-3, 2000, pp. 83-88. doi:10.1016/S0375-9601(00)00252-8

[8] S. A. S. Ahmed, "A Transformation Method of Generating Exact Analytic Solutions of the Schrödinger Equation," International Journal of Theoretical Physics, Vol. 36, No. 8, 1997, pp. 1893-1905. doi:10.1007/BF02435851

[9] C. Chen, C. Liu and F. Lu, "Exact Solutions of Schrödinger Equation for the Makarov Potential," Physics Letters A, Vol. 374, No. 11-12, 2010, pp. 1346-1349. doi:10.1016/i.physleta.2010.01.018

[10] M. Abramowitz and I. A. Stegun, "Handbook of Mathematical Functions," Dover Publications, New York, 1978.

[11] D. E. Alvarez-Castillo and M. Kirchbach, "Exact Spectrum and Wave Functions of the Hyperbolic Scarf Potential in Terms of Finite Romanovski Polynomials," Revista Mexicana de Fisica, Vol. E53, No. 2, 2007, pp. 143-154. 